\section{(1) \\ ИНФОРМАТИКА \\ УДК 517.935.2 \\ УСЛОВИЯ АНАЛИТИЧНОСТИ ХАРАКТЕРИСТИЧЕСКОГО И ВОЗМУЩАЮЩИХ КВАЗИМНОГОЧЛЕНОВ КОМБИНИРОВАННЫХ ДИНАМИЧЕСКИХ СИСТЕМ}

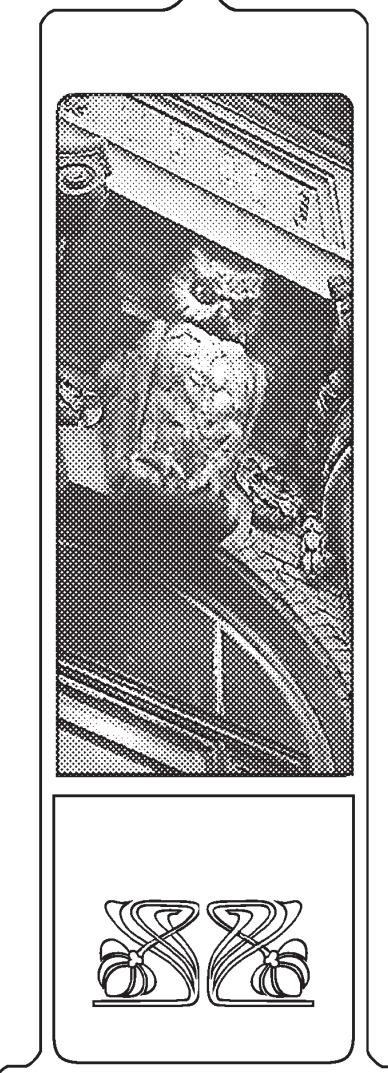

НАУЧНЫЙ

OTAEN

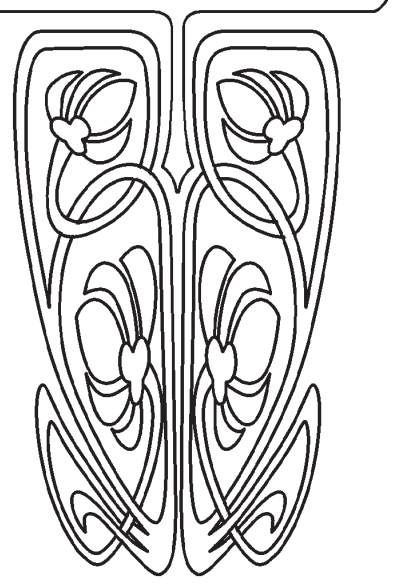

\author{
М. С. Портенко ${ }^{1}$, Д. В. Мельничук ${ }^{2}$, Д. К. Андрейченко ${ }^{3}$
}

\begin{abstract}
${ }^{1}$ Портенко Марина Сергеевна, старший преподаватель кафредры инфрорматики и программирования, Саратовский национальный исследовательский государственный университет им. Н. Г. Чернышевского, msportenko@gmail.com

${ }^{2}$ Мельничук Дмитрий Вадимович, магистрант фракультета компьютерных наук и инсрормационных технологий, Саратовский национальный исследовательский государственный университет им. Н. Г. Чернышевского, meldm007@gmail.com

${ }^{3}$ Андрейченко Дмитрий Констатинович, доктор фризико-математических наук, просрессор кафредры математического обеспечения вычислительных комплексов и инсрормационных систем, Саратовский национальный исследовательский государственный университет им. Н. Г. Чернышевского, kp_andreichenko@ renet.ru

Комбинированные динамические системы (КДС) представляют собой связанные посредством граничных условий и условий связи системы обыкновенных дисрфреренциальных уравнений и уравнений в частных производных при соответствующих начальных условиях. Проверка устойчивости КДС может быть выполнена на основе быстрого алгоритма, для применения которого необходима аналитичность характеристического и возмущающих квазимногочленов КДС в правой комплексной полуплоскости и вблизи мнимой оси. В статье ссрормулированы и доказаны условия аналитичности характеристического и возмущающих квазимногочленов КДС. Рассмотрены математические модели объектов управления с распределенными по пространству параметрами, соответствущие процессам теплопроводности и дисрфузии, динамики поддерживающих слоев вязкой несжимаемой жидкости, а также динамики упругодесрормируемой среды с учетом внутреннего трения.
\end{abstract}

Ключевые слова: комбинированные динамические системы, устойчивость.

DOI: 10.18500/1816-9791-2016-16-2-208-217

\section{ВВЕДЕНИЕ}

Комбинированные динамические системы (КДС) - это математические модели в форме связанных посредством граничных условий и условий связи обыкновенных дифференциальных уравнений и уравнений в частных производных при соответствующих начальных условиях $[1,2]$. Основные теоремы об устойчивости КДС сформулированы и доказаны в $[1,2]$, где приведен основанный на теореме об устойчивом квазимногочлене «быстрый» алгоритм проверки устойчивости КДС. Для его применения необходимо, чтобы характеристический и возмущающий квазимногочлены КДС были аналитическими функциями в правой комплексной полуплоскости и вблизи мнимой оси. В общем случае вопрос об условиях аналитичности характеристического и возмущающего квазимногочленов КДС остался открыт, а его решение является целью данной работы. 


\section{1. КОМБИНИРОВАННЫЕ ДИНАМИЧЕСКИЕ СИСТЕМЫ}

Структурная схема КДС с входной вектор-функцией $\mathbf{x}: \mathbb{R} \rightarrow \mathbb{R}^{N_{x}}$ и характеризующей движение объектов управления с сосредоточенными по пространству параметрами выходной вектор-функцией $\mathbf{y}: \mathbb{R} \rightarrow \mathbb{R}^{N_{y}}$ приведена на рис. 1 (где $t-$ время). Здесь ОДУ - обыкновенные дифференциальные уравнения, УЧП - уравнения в частных производных, ГУ - граничные условия, УС - условия связи, НУ - начальные условия. Модельные уравнения движения КДС можно привести к виду $[2$, с. 8]

$$
\begin{gathered}
\dot{\mathbf{y}}=\mathbf{f}(\mathbf{x}, \mathbf{y}, \mathbf{h}) ; \quad \dot{\mathbf{u}}=\mathbb{F}(\mathbf{u}, \mathbf{x}, \mathbf{y}, \dot{\mathbf{y}}), \quad \mathbf{r} \in \Omega \subset \mathbb{R}^{N_{r}}, \\
\left.\mathbb{G}(\mathbf{u}, \mathbf{y})\right|_{S}=0 ; \quad \mathbf{h}=\int_{S} \mathbb{H}(\mathbf{u}) d S, \quad S=\partial \Omega, \\
\mathbf{y}(0)=\mathbf{y}_{0}, \quad \mathbf{u}(\mathbf{r}, 0)=\mathbf{u}_{0}(\mathbf{r}) .
\end{gathered}
$$

Здесь $\mathbf{r}-$ независимые пространственные координаты; $\Omega$, $S$ - область, занимаемая объектами управления с распределенными по пространству параметрами и ее граница соответственно; $\mathbf{u}: \mathbb{R}^{N_{r}} \times \mathbb{R} \rightarrow \mathbb{R}^{N_{u}}$ характеризует движение объектов управления с распределенными по пространству

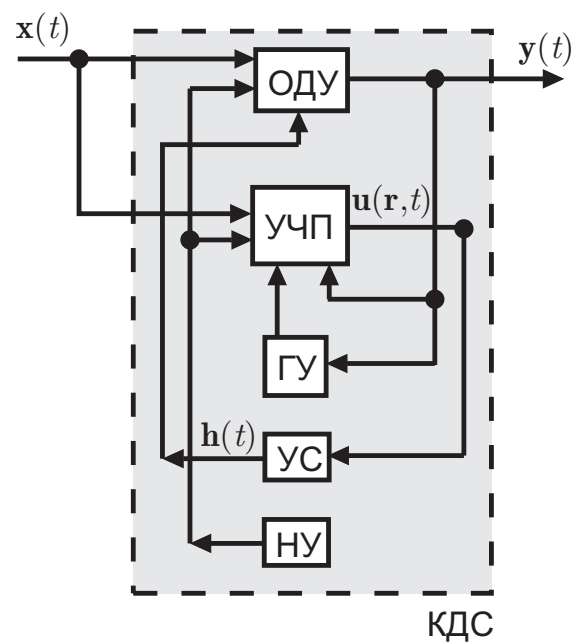

Рис. 1. Структурная схема КДС параметрами; $\mathbf{h}: \mathbb{R} \rightarrow \mathbb{R}^{N_{h}} ; \mathbf{f}: \mathbb{R}^{N_{x}} \times \mathbb{R}^{N_{y}} \times \mathbb{R}^{N_{h}} \rightarrow \mathbb{R}^{N_{y}} ;(:)=d(\cdot) / d t$ либо $(:)=\partial(\cdot) / \partial t ;$ операторы $\mathbb{F}, \mathbb{G}, \mathbb{H}$ соответствуют уравнениям в частных производных, граничным условиям и условиям связи. Предполагается, что вектор-функция $\mathbf{f}$ и операторы $\mathbb{F}, \mathbb{G}, \mathbb{H}$ не зависят от времени $t$, т.е. система является стационарной с точки зрения теории управления. В равновесном состоянии $\mathbf{x}=\mathbf{x}_{0}=$ const, $(:)=0$, и характеризующие его величины $\mathbf{y}_{0}, \mathbf{u}_{0}(\mathbf{r}), \mathbf{h}_{0}$ суть решение уравнений

$$
\begin{aligned}
& \mathbf{f}(\mathbf{x}, \mathbf{y}, \mathbf{h})=0 ; \quad \mathbf{h}=\int_{S} \mathbb{H}(\mathbf{u}) d S, \\
& \mathbb{F}(\mathbf{u}, \mathbf{x}, \mathbf{y}, 0)=0, \quad \mathbf{r} \in \Omega ;\left.\quad \mathbb{G}(\mathbf{u}, \mathbf{y})\right|_{S}=0 .
\end{aligned}
$$

В предположении $\mathbf{x}(t)=\mathbf{x}_{0}+\mu \mathbf{x}^{*}(t), \mathbf{y}(t)=\mathbf{y}_{0}+\mu \mathbf{y}^{*}(t), \quad \mathbf{u}(\mathbf{r}, t)=\mathbf{u}_{0}(\mathbf{r})+\mu \mathbf{u}^{*}(\mathbf{r}, t)$, $\mathbf{h}(t)=\mathbf{h}_{0}+\mu \mathbf{h}^{*}(t), \mu \rightarrow 0$, при условии дифференцируемости функции $\mathbf{f}$ и операторов $\mathbb{F}, \mathbb{G}, \mathbb{H}$ из (1)-(3) следуют уравнения относительно величин $(\cdot)^{*}$, характеризующих возмущенное движение (символ * далее опущен), которые после выполнения интегрального преобразования Лапласа по времени $f(t) \rightarrow \tilde{f}(\lambda)=\int_{0}^{\infty} f(t) e^{-\lambda t} d t$ принимают вид

$$
\begin{gathered}
\lambda \tilde{\mathbf{y}}=B \tilde{\mathbf{x}}+C \tilde{\mathbf{y}}+A \tilde{\mathbf{h}}, \\
\lambda \tilde{\mathbf{u}}=\mathbb{L}_{1}^{(F)}(\tilde{\mathbf{u}})+L_{2}^{(F)} \tilde{\mathbf{x}}+\left(L_{3}^{(F)}+\lambda L_{4}^{(F)}\right) \tilde{\mathbf{y}}, \quad \mathbf{r} \in \Omega ;\left.\quad\left(\mathbb{L}_{1}^{(G)}(\tilde{\mathbf{u}})+L_{2}^{(G)} \tilde{\mathbf{y}}\right)\right|_{S}=0, \\
\tilde{\mathbf{h}}=\int_{S} \mathbb{L}^{(H)}(\tilde{\mathbf{u}}) d S .
\end{gathered}
$$

Здесь $A, B, C$ - постоянные матрицы; $L_{2}^{(F)}, L_{3}^{(F)}, L_{4}^{(F)}, L_{2}^{(G)}$ - матрицы, которые могут зависеть от $\mathbf{r}$; линейные операторы $\mathbb{L}_{1}^{(F)}, \mathbb{L}_{1}^{(G)}, \mathbb{L}^{(H)}$ соответствуют линеаризованным уравнениям в частных производных, граничным условиям и условиям связи. Из линейности по $\tilde{\mathbf{u}}, \tilde{\mathbf{x}}, \tilde{\mathbf{y}}$ краевой задачи (5) и линейности (6) по и̃ следует

$$
\tilde{\mathbf{h}}(\lambda)=B_{u}(\lambda) \tilde{\mathbf{x}}(\lambda)+C_{u}(\lambda) \tilde{\mathbf{y}}(\lambda), \quad B_{u}: \mathbb{C} \rightarrow \mathbb{C}^{\left(N_{h}, N_{x}\right)}, \quad C_{u}: \mathbb{C} \rightarrow \mathbb{C}^{\left(N_{h}, N_{y}\right)},
$$

причем столбцы с номером $j$ матриц $B_{u}(\lambda)$ и $C_{u}(\lambda)$ находятся при помощи решения линейных краевых задач относительно вспомогательной функции $\mathbf{v}(\mathbf{r}, \lambda), \mathbf{v}: \Omega \times \mathbb{C} \rightarrow \mathbb{C}^{N_{u}}$

$$
\begin{gathered}
\lambda \mathbf{v}=\mathbb{L}_{1}^{(F)}(\mathbf{v})+L_{2}^{(F)} \mathbf{e}_{j}^{\left(N_{x}\right)}, \quad \mathbf{r} \in \Omega,\left.\quad \mathbb{L}_{1}^{(G)}(\mathbf{v})\right|_{S}=0, \\
B_{u}(\lambda) \mathbf{e}_{j}^{\left(N_{x}\right)}=\int_{S} \mathbb{L}^{(H)}(\mathbf{v}) d S, \quad j=\overline{1, N_{x}},
\end{gathered}
$$




$$
\begin{array}{cl}
\lambda \mathbf{v}=\mathbb{L}_{1}^{(F)}(\mathbf{v})+\left(L_{3}^{(F)}+\lambda L_{4}^{(F)}\right) \mathbf{e}_{j}^{\left(N_{y}\right)}, \quad \mathbf{r} \in \Omega ; & \left.\left(\mathbb{L}_{1}^{(G)}(\mathbf{v})+L_{2}^{(G)} \mathbf{e}_{j}^{\left(N_{y}\right)}\right)\right|_{S}=0, \\
C_{u}(\lambda) \mathbf{e}_{j}^{\left(N_{y}\right)}=\int_{S} \mathbb{L}^{(H)}(\mathbf{v}) d S, & j=\overline{1, N_{y}},
\end{array}
$$

где $\mathbf{e}_{j}^{(N)} \in \mathbb{R}^{N}, j=\overline{1, N} ; \mathbf{e}_{1}^{(N)}=(1,0, \ldots 0)^{T}, \mathbf{e}_{2}^{(N)}=(0,1, \ldots 0)^{T}, \ldots, \mathbf{e}_{N}^{(N)}=(0,0, \ldots 1)^{T}$. Подстановка (7) в (4) приводит к системе линейных уравнений, связывающей $\tilde{\mathbf{x}}(\lambda)$ и $\tilde{\mathbf{y}}(\lambda)$, из которой находится матрица передаточных функций $\Phi(\lambda)$ и выражения для характеристического квазимногочлена $D(\lambda)$ и возмущающих квазимногочленов $Q_{k j}(\lambda)$

$$
\begin{gathered}
\tilde{\mathbf{y}}(\lambda)=\Phi(\lambda) \tilde{\mathbf{x}}(\lambda), \\
\Phi(\lambda)=\left[Q_{k j}(\lambda) / D(\lambda)\right]=\left[\lambda E-C-A C_{u}(\lambda)\right]^{-1}\left[B+A B_{u}(\lambda)\right], \quad \\
D(\lambda)=\operatorname{det}\left[\lambda E-C-A C_{u}(\lambda)\right], \quad E=\operatorname{diag}\{1,1, \ldots, 1\}, \quad k=\overline{1, N_{y}}, \quad j=\overline{1, N_{x}} .
\end{gathered}
$$

Так как краевые задачи (8), (9) зависят лишь от одной комплексной величины $\lambda$

$$
\begin{gathered}
B_{u}(\bar{\lambda})=\overline{B_{u}(\lambda)}, \quad C_{u}(\bar{\lambda})=\overline{C_{u}(\lambda)}, \\
D(\bar{\lambda})=\overline{D(\lambda)}, \quad Q_{k j}(\bar{\lambda})=\overline{Q_{k j}(\lambda)}, \quad k=\overline{1, N_{y}}, \quad j=\overline{1, N_{x}} .
\end{gathered}
$$

Обобщенная степень $n \in \mathbb{R}$ (как правило, $n=N_{u}$ ) характеристичекого квазимногочлена $D(\lambda)$ находится из условия $[1,2]$

$$
\lim _{\lambda \rightarrow \infty} \lambda^{-n} D(\lambda)=c_{a}, \quad 0<\left|c_{a}\right|<\infty, \quad \operatorname{Re} \lambda>\sigma_{0}, \quad \sigma_{0} \in(-\infty, 0) .
$$

При условии аналитичности функций $D(\lambda)$ и $Q_{k j}(\lambda)$ при $\operatorname{Re} \lambda>\sigma_{0}, \sigma_{0} \in(-\infty, 0)$, наличия обобщенной степени (13) и некоторых других условий из теорем об устойчивости КДС [1,2] (об устойчивом квазимногочлене) следует критерий устойчивости КДС

$$
\underset{0 \leqslant \omega<\infty}{\Delta} \arg D(i \omega)=n \pi / 2 .
$$

При оптимизации шага изменения $\omega$ условие (14) представляет собой «быстрый» алгоритм проверки устойчивости КДС.

\section{2. ПРОЕКЦИОННЫЙ МЕТОД ГАЛЕРКИНА}

Данный метод применяется для численного решения нелинейной краевой задачи (2), (3). Пусть $\mathbf{W}_{k}(\mathbf{r}), \mathbf{W}_{k}: \Omega \rightarrow \mathbb{R}^{N_{u}} \quad k=1,2, \ldots-$ полная система функций в области $\Omega ; \Gamma_{k}\left(\left.\mathbf{r}\right|_{s}\right), \Gamma_{k}: S \rightarrow \mathbb{R}^{N_{G}}$, $k=1,2, \ldots-$ полная система функций на $S=\partial \Omega$. Полагаем

$$
\mathbf{u}(\mathbf{r}) \approx \sum_{k=1}^{N_{\Omega}+N_{S}} u_{k} \mathbf{W}_{k}(\mathbf{r})
$$

и для приближенного выполнения уравнений (3) требуем

$$
\int_{\Omega} \mathbb{F}(\mathbf{u}, \mathbf{x}, \mathbf{y}, 0) \cdot \mathbf{W}_{k}(\mathbf{r}) d \Omega=0, \quad k=\overline{1, N_{\Omega}} ; \quad \int_{S} \mathbb{G}(\mathbf{u}, \mathbf{y}) \cdot \Gamma_{k}(\mathbf{r}) d S=0, \quad k=\overline{1, N_{S}},
$$

где (.).(·) - скалярное произведение векторов соответстветствующей размерности. Из (3), (16) следует система нелинейных уравнений относительно компонент вектора $\mathbf{Y}$

$$
\begin{gathered}
\mathbf{F}(\mathbf{Y})=0, \quad \mathbf{F}: \mathbb{R}^{N_{y}+N_{\Omega}+N_{S}} \rightarrow \mathbb{R}^{N_{y}+N_{\Omega}+N_{S}}, \\
\mathbf{Y}=\left(y_{1}, y_{2}, \ldots, y_{N_{y}}, u_{1}, u_{2}, \ldots, u_{N_{\Omega}+N_{S}}\right)^{T} \in \mathbb{R}^{N_{y}+N_{\Omega}+N_{S}} .
\end{gathered}
$$

Далее система нелинейных уравнений (17) решается численно.

Проекционный метод Галеркина также применяется для решения линейных краевых задач (8), (9), при этом достаточно рассмотреть (9). Полагаем

$$
\mathbf{v}(\mathbf{r}, \lambda) \approx \sum_{k=1}^{N_{\Omega}+N_{S}} v_{k}(\lambda) \mathbf{W}_{k}(\mathbf{r}) .
$$


Дискретный аналог (9) принимает вид

$$
\begin{gathered}
\lambda \int_{\Omega} \mathbf{v} \cdot \mathbf{W}_{k}(\mathbf{r}) d \Omega=\int_{\Omega}\left[\mathbb{L}_{1}^{(F)}(\mathbf{v})+\left(L_{3}^{(F)}+\lambda L_{4}^{(F)}\right) \mathbf{e}_{j}^{\left(N_{y}\right)}\right] \cdot \mathbf{W}_{k}(\mathbf{r}) d \Omega, \quad k=\overline{1, N_{\Omega}}, \\
\int_{S} \mathbb{L}_{1}^{(G)}(\mathbf{v}) \cdot \Gamma_{k}(\mathbf{r}) d S=-\int_{S} L_{2}^{(G)} \mathbf{e}_{j}^{\left(N_{y}\right)} \cdot \Gamma_{k}(\mathbf{r}) d S, \quad k=\overline{1, N_{S}} .
\end{gathered}
$$

Подстановка (18) в (19) приводит к системе линейных уравнений размерности $\left(N_{\Omega}+N_{S}\right) \times$ $\times\left(N_{\Omega}+N_{S}\right)$ относительно коэффициентов разложения (18), которая решается численно, и далее используются формулы (10) для вычисления текущего столбца матрицы $C_{u}(\lambda)$. Матрица коэффициентов и правые части системы линейных уравнений (16) линейно зависят от параметра $\lambda$. Пусть $\mathscr{D}(\lambda)$ - определитель системы линейных уравнений (16), являющийся полиномом некоторой степени параметра $\lambda$. Из (10) следует, что в области, где применяется проекционный метод Галеркина, особенности элементов вспомогательной матрицы $C_{u}(\lambda)$ (а также $B_{u}(\lambda)$ и, в конечном счете, характеристического и возмущающего квазимногочленов $D(\lambda)$ и $\left.Q_{k j}(\lambda)\right)$ будут исчерпываться особенностями решения системы линейных уравнений (19), т.е. нулями определителя $\mathscr{D}(\lambda)$. Проверка отсутствия нулей $\mathscr{D}(\lambda)$ в области, ограниченной контуром

$$
\begin{gathered}
\ell_{R}=\left\{\lambda=-\sigma_{0}-i \omega,-\left(R^{2}-\sigma_{0}^{2}\right)^{1 / 2} \leqslant \omega \leqslant\left(R^{2}-\sigma_{0}^{2}\right)^{1 / 2}\right\} \cup \\
\cup\left\{\lambda=R e^{i \vartheta},-\left[\pi / 2+\arcsin \left(\sigma_{0} / R\right)\right] \leqslant \vartheta \leqslant \pi / 2+\arcsin \left(\sigma_{0} / R\right)\right\}, \\
R \gg 1, \quad 0<\sigma_{0} \ll 1,
\end{gathered}
$$

на основании принципа аргумента сводится к проверке условия (рис. 2)

$$
\underset{\lambda \in \ell_{R}}{\Delta} \arg \mathscr{D}(\lambda)=0 .
$$

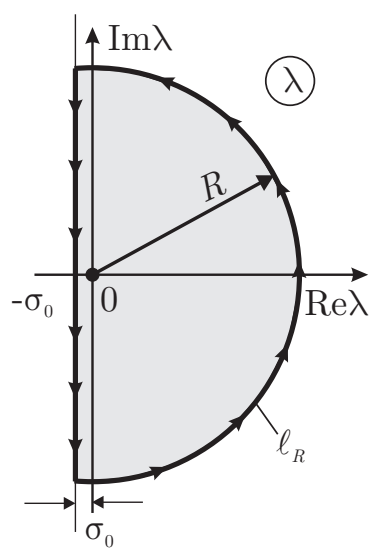

\section{3. УСЛОВИЯ АНАЛИТИЧНОСТИ}

При фиксированном $\lambda$ в линейном пространстве функций $\mathbf{u}: \Omega \rightarrow \mathbb{C}^{N_{u}}$ введем норму $\|\mathbf{u}\| \in \mathbb{R}$, например, $\|\mathbf{u}\|=\left[\int_{\Omega}|\mathbf{u}|^{2} d \Omega\right]^{1 / 2}$. Полагаем, что в (8), (9)

$$
\left\|L_{2}^{(F)} \mathbf{e}_{j}^{\left(N_{x}\right)}\right\|<\infty, \quad j=\overline{1, N_{x}}, \quad\left\|L_{3}^{(F)} \mathbf{e}_{j}^{\left(N_{y}\right)}\right\|<\infty, \quad\left\|L_{4}^{(F)} \mathbf{e}_{j}^{\left(N_{y}\right)}\right\|<\infty, \quad j=\overline{1, N_{y}} .
$$

Так как входящий в уравнения в частных производных неограниченный линейный оператор $\mathbb{L}_{1}^{(F)}$ содержит производные более высокого порядка, чем входящий в условия связи линейный оператор $\mathbb{L}^{(H)}$, для достаточно гладких функций полагаем

$$
\forall\left(\mathbf{u}: \Omega \rightarrow \mathbb{C}^{N_{u}}\right): \quad\left\|\mathbb{L}_{1}^{(F)}(\mathbf{u})\right\|<\infty,\left.\quad \mathbb{L}^{(G)}(\mathbf{u})\right|_{S}=0 \quad \Rightarrow \quad\left|\int_{S} \mathbb{L}^{(H)}(\mathbf{u}) d S\right|<\infty .
$$

Далее полагаем: существуют достаточно гладкие функции $\mathbf{v}_{0}^{(j)}: \Omega \rightarrow \mathbb{R}^{N_{u}}$ такие, что

$$
\begin{gathered}
\left\|\mathbf{v}_{0}^{(j)}\right\|<\infty,\left.\quad \mathbb{L}_{1}^{(G)}\left(\mathbf{v}_{0}^{(j)}\right)\right|_{S}=-L_{2}^{(G)} \mathbf{e}_{j}^{\left(N_{y}\right)}, \quad\left\|\mathbb{L}_{1}^{(F)}\left(\mathbf{v}_{0}^{(j)}\right)\right\|<\infty, \\
\mathbf{c}_{u}^{(j)}=\int_{S} \mathbb{L}^{(H)}\left(\mathbf{v}_{0}^{(j)}\right) d S, \quad\left|\mathbf{c}_{u}^{(j)}\right|<\infty, \quad j=\overline{1, N_{y}} .
\end{gathered}
$$

Лемма 1. Пусть выполнены условия (22)-(24) и в некоторой области комплексной плоскости при $\lambda \in \Omega_{\lambda} \subset \mathbb{C}$ для функций (23) справедливо неравенство

$$
\left\|\lambda \mathbf{u}-\mathbb{L}_{1}^{(F)}(\mathbf{u})\right\| \geqslant m(\lambda)\|\mathbf{u}\|, \quad m(\lambda)>0 .
$$

Тогда при $\lambda \in \Omega_{\lambda}$ элементы матриц $B_{u}(\lambda)$ и $C_{u}(\lambda)$ - комплекснозначные однозначные ограниченные функции параметра $\lambda$. 
Доказательство. Достаточно рассмотреть вспомогательную краевую задачу (9) и формулы (10), которые, используя (24) и полагая $\mathbf{v}=\mathbf{v}_{0}+\mathbf{w}$, преобразуем к виду

$$
\begin{gathered}
\lambda \mathbf{w}=\mathbb{L}_{1}^{(F)}(\mathbf{w})+\mathbf{f}_{0}(\mathbf{r})+\lambda \mathbf{f}_{1}(\mathbf{r}), \quad \mathbf{r} \in \Omega ;\left.\quad \mathbb{L}_{1}^{(G)}(\mathbf{w})\right|_{S}=0, \\
\mathbf{f}_{0}(\mathbf{r})=\mathbb{L}_{1}^{(F)}\left(\mathbf{v}_{0}\right)+L_{3}^{(F)} \mathbf{e}_{j}^{\left(N_{y}\right)}, \quad \mathbf{f}_{1}(\mathbf{r})=L_{4}^{(F)} \mathbf{e}_{j}^{\left(N_{y}\right)}-\mathbf{v}_{0}, \\
C_{u}(\lambda) \mathbf{e}_{j}^{\left(N_{y}\right)}=\mathbf{c}_{u}^{(j)}+\int_{S} \mathbb{L}^{(H)}(\mathbf{w}) d S, \quad j=\overline{1, N_{y}} .
\end{gathered}
$$

Оператор $\lambda()-.\mathbb{L}_{1}^{(F)}($.$) отображает линейное нормированное пространство достаточно гладких$ удовлетворяющих однородным граничным условиям функций (23) в линейное нормированное пространство ограниченных по норме функций. Как следует из (25), согласно [3, с. 119, теорема 1] оператор $\lambda()-.\mathbb{L}_{1}^{(F)}($.$) при \lambda \in \Omega_{\lambda}$ имеет ограниченный обратный оператор, т. е. с учетом (22)-(24) существует единственное достаточно гладкое решение вспомогательной краевой задачи, для которого существует и ограничен интеграл в правой части равенства (27).

Лемма 2. Пусть выполнены условия (22)-(24) и в некоторой замкнутой области комплексной плоскости при $\lambda \in \bar{\Omega}_{\lambda}=\Omega_{\lambda} \cup \partial \Omega_{\lambda} \subset \mathbb{C}$ для функциий (23) спарведливо условие (25). Тогда при $\lambda \in \Omega_{\lambda}$ әлементы матрии $B_{u}(\lambda)$ и $C_{u}(\lambda)$ - аналитические функции $\lambda$.

Доказательство. Достаточно рассмотреть вспомогательную краевую задачу (9) и формулы (10). Зафиксируем $\lambda_{0} \in \Omega_{\lambda}$ и некоторое $\lambda \in \bar{\Omega}_{\lambda}$. Используя линейность (26) и (27) и полагая $\mathbf{w}(\mathbf{r}, \lambda)=\mathbf{u}_{0}(\mathbf{r}$, $\left.\lambda_{0}\right)+\left(\lambda-\lambda_{0}\right) \mathbf{u}_{1}\left(\mathbf{r}, \lambda_{0}\right)+\left(\lambda-\lambda_{0}\right)^{2} \mathbf{u}_{2}\left(\mathbf{r}, \lambda_{0}, \lambda\right)$, находим

$$
\begin{gathered}
\lambda_{0} \mathbf{u}_{0}=\mathbb{L}_{1}^{(F)}\left(\mathbf{u}_{0}\right)+\mathbf{f}_{0}(\mathbf{r})+\lambda_{0} \mathbf{f}_{1}(\mathbf{r}), \quad \mathbf{r} \in \Omega ;\left.\quad \mathbb{L}_{1}^{(G)}\left(\mathbf{u}_{0}\right)\right|_{S}=0, \\
\lambda_{0} \mathbf{u}_{1}=\mathbb{L}_{1}^{(F)}\left(\mathbf{u}_{1}\right)+\mathbf{f}_{1}(\mathbf{r})-\mathbf{u}_{0}, \quad \mathbf{r} \in \Omega ;\left.\quad \mathbb{L}_{1}^{(G)}\left(\mathbf{u}_{1}\right)\right|_{S}=0, \\
\lambda \mathbf{u}_{2}=\mathbb{L}_{1}^{(F)}\left(\mathbf{u}_{2}\right)-\mathbf{u}_{1}, \quad \mathbf{r} \in \Omega ;\left.\quad \mathbb{L}_{1}^{(G)}\left(\mathbf{u}_{2}\right)\right|_{S}=0, \\
C_{u}(\lambda) \mathbf{e}_{j}^{\left(N_{y}\right)}=\mathbf{c}_{u}^{(j)}+\mathbf{g}_{0}^{(j)}\left(\lambda_{0}\right)+\mathbf{g}_{1}^{(j)}\left(\lambda_{0}\right)\left(\lambda-\lambda_{0}\right)+\mathbf{g}_{2}^{(j)}\left(\lambda_{0}, \lambda\right)\left(\lambda-\lambda_{0}\right)^{2}, \\
\mathbf{g}_{0}^{(j)}\left(\lambda_{0}\right)=\int_{S} \mathbb{L}^{(H)}\left(\mathbf{u}_{0}\right) d S, \quad \mathbf{g}_{1}^{(j)}\left(\lambda_{0}\right)=\int_{S} \mathbb{L}^{(H)}\left(\mathbf{u}_{1}\right) d S, \quad \mathbf{g}_{2}^{(j)}\left(\lambda_{0}, \lambda\right)=\int_{S} \mathbb{L}^{(H)}\left(\mathbf{u}_{2}\right) d S, \quad j=\overline{1, N_{y} .}
\end{gathered}
$$

Аналогично доказательству леммы 1 можно показать: при $\lambda_{0} \in \Omega_{\lambda} \mathbf{g}_{0}^{(j)}\left(\lambda_{0}\right), \mathbf{g}_{1}^{(j)}\left(\lambda_{0}\right)$ - ограниченные комплекснозначные вектор-функции $\lambda_{0}$; при $\lambda_{0} \in \Omega_{\lambda}, \lambda \in \bar{\Omega}_{\lambda} \mathbf{g}_{2}^{(j)}\left(\lambda_{0}, \lambda\right)$ - ограниченная комплекснозначная вектор-функция своих аргументов. Так как в замкнутой области $\bar{\Omega}_{\lambda}$ можно найти $M\left(\lambda_{0}\right)=\max _{\lambda \in \bar{\Omega}_{\lambda}}\left|\mathbf{g}_{2}^{(j)}\left(\lambda_{0}, \lambda\right)\right|$, это означает наличие производной элементов матрицы $C_{u}(\lambda)$ в точке $\lambda_{0} \in \Omega_{\lambda}$, т. е. их аналитичность в области $\Omega_{\lambda}$.

Теорема 1. Пусть выполнены условия (22)-(24) и в некоторой замкнутой области комплексной плоскости при $\lambda \in \bar{\Omega}_{\lambda}=\Omega_{\lambda} \cup \partial \Omega_{\lambda} \subset \mathbb{C}$ для функций (23) справедиво условие (25). Тогда при $\lambda \in \Omega_{\lambda}$ характеристический квазимногочлен $D(\lambda)$ и возмущающие квазимногочлены $Q_{k j}(\lambda)$, $k=\overline{1, N_{y}}, j=\overline{1, N_{x}},-$ аналитические функции $\lambda$.

Доказательство. Непосредственно следует из (11) и леммы 2.

\section{4. АНАЛИТИЧНОСТЬ В ВЫСОКОЧАСТОТНОЙ ОБЛАСТИ}

В «низкочастотной» области внутри контура (20) (см. рис. 2) проверка аналитичности характеристического и возмущающих квазимногочленов эффективно реализуется на основе условия (21). Аналитичность характеристического и возмущающих квазимногочленов в высокочастотной области $\lambda \rightarrow \infty, \operatorname{Re} \lambda>-\infty$ устанавливается на основе теоремы 1 . При фиксированном $\lambda$ для функций $\mathbf{u}, \mathbf{v}: \Omega \rightarrow \mathbb{C}^{N_{u}}$ введем операцию скалярного произведения, например $\langle\mathbf{u}, \mathbf{v}\rangle=\int_{\Omega} \mathbf{u} \cdot \mathbf{v} d \Omega$, $\langle\mathbf{v}, \mathbf{u}\rangle=\overline{\langle\mathbf{u}, \mathbf{v}\rangle},\|\mathbf{u}\|=\sqrt{\langle\mathbf{u}, \mathbf{u}\rangle}$. Пусть уравнения типа (1) моделируют процессы типа теплопроводности или диффузии, тогда

$$
\mathbb{L}_{1}^{(F)}=\mathbb{L}_{v, 1}^{(F)}-\mathbb{L}_{v, 2}^{(F)} .
$$


Линейный оператор $\mathbb{L}_{v, 1}^{(F)}$ может быть ограниченным либо неограниченным. Линейный оператор $\mathbb{L}_{v, 2}^{(F)}$ содержит производные наивысшего порядка по независимым пространственным переменным, неограничен, положительно определен и самосопряжен

$$
\begin{aligned}
& \forall\left(\mathbf{u}: \Omega \rightarrow \mathbb{R}^{N_{u}}\right): \quad \mathbf{u} \neq \mathbf{0},\left.\quad \mathbb{L}_{1}^{(G)}(\mathbf{u})\right|_{S}=0 \quad \Rightarrow \quad\left\langle\mathbf{u}, \mathbb{L}_{v, 2}^{(F)}(\mathbf{u})\right\rangle>0 \\
& \forall\left(\mathbf{u}, \mathbf{v}: \Omega \rightarrow \mathbb{R}^{N_{u}}\right):\left.\quad \mathbb{L}_{1}^{(G)}(\mathbf{u})\right|_{S}=0,\left.\mathbb{L}_{1}^{(G)}(\mathbf{v})\right|_{S}=0 \quad \Rightarrow\left\langle\mathbf{v}, \mathbb{L}_{v, 2}^{(F)}(\mathbf{u})\right\rangle=\left\langle\mathbf{u}, \mathbb{L}_{v, 2}^{(F)}(\mathbf{v})\right\rangle
\end{aligned}
$$

Поскольку операторы $\mathbb{L}_{v, 2}^{(F)}$ и $\mathbb{L}_{1}^{(G)}$ являются вещественными, из (29) следует

$$
\forall\left(\mathbf{u}: \Omega \rightarrow \mathbb{C}^{N_{u}}\right): \quad\|\mathbf{u}\|=1,\left.\quad \mathbb{L}_{1}^{(G)}(\mathbf{u})\right|_{S}=0 \quad \Rightarrow \quad\left\langle\mathbf{u}, \mathbb{L}_{v, 2}^{(F)}(\mathbf{u})\right\rangle>0 .
$$

Так как оператор $\mathbb{L}_{v, 2}^{(F)}$ содержит производные наивысшего порядка, полагаем

$$
\begin{gathered}
\forall\left(\mathbf{u}: \Omega \rightarrow \mathbb{C}^{N_{u}}\right): \quad\|\mathbf{u}\|=1,\left.\quad \mathbb{L}_{1}^{(G)}(\mathbf{u})\right|_{S}=0, \quad\left\|\mathbb{L}_{v, 2}^{(F)}(\mathbf{u})\right\| \gg 1 \quad \Rightarrow \\
\Rightarrow \quad\left|\left\langle\mathbf{u}, \mathbb{L}_{v, 1}^{(F)}(\mathbf{u})\right\rangle\right| \ll\left\langle\mathbf{u}, \mathbb{L}_{v, 2}^{(F)}(\mathbf{u})\right\rangle .
\end{gathered}
$$

Теорема 2. Пусть выполнень условия (22)-(24), (28), (29) и (31). Тогда при $|\lambda| \gg 1$, $\operatorname{Re} \lambda>-|\lambda| \sin \alpha, 0<\alpha<\pi / 2$ характеристический квазимногочлен $D(\lambda)$ и возмущающие квазимногочлены $Q_{k j}(\lambda), k=\overline{1, N_{y}}, j=\overline{1, N_{x}}$, - аналитические функции $\lambda$.

Доказательство. Достаточно рассмотреть вспомогательную краевую задачу (26) и формулы (27). Как следует из (26), для решения данной вспомогательной линейной краевой задачи при $\lambda \rightarrow \infty$, $\operatorname{Re} \lambda>-\infty\|\mathbf{w}\|<M<\infty \Rightarrow\left\|\mathbb{L}_{v, 2}^{(F)}(\mathbf{w})\right\| \rightarrow \infty$. В таком случае, как следует из (30), (31), при $\lambda \rightarrow \infty, 0<\alpha<\pi / 2$, $\operatorname{Re} \lambda>-|\lambda| \sin \alpha$ (рис. 3)

$$
\begin{gathered}
\forall\left(\mathbf{u}: \Omega \rightarrow \mathbb{C}^{N_{u}}\right): \quad\|\mathbf{u}\|=1,\left.\quad \mathbb{L}_{1}^{(G)}(\mathbf{u})\right|_{S}=0 \quad \Rightarrow \\
\Rightarrow \quad\left\|\lambda \mathbf{u}-\mathbb{L}_{1}^{(F)}(\mathbf{u})\right\| \geqslant\left|\left\langle\mathbf{u}, \lambda \mathbf{u}-\mathbb{L}_{1}^{(F)}(\mathbf{u})\right\rangle\right|= \\
=\left|\lambda+\left\langle\mathbf{u},-\mathbb{L}_{1}^{(F)}(\mathbf{u})\right\rangle\right| \geqslant \frac{1}{2}\left|\lambda+\left\langle\mathbf{u}, \mathbb{L}_{v, 2}^{(F)}(\mathbf{u})\right\rangle\right| \geqslant \frac{1}{2}|\lambda| \cos \alpha \\
\forall\left(\mathbf{u}: \Omega \rightarrow \mathbb{C}^{N_{u}}\right):\left.\quad \mathbb{L}_{1}^{(G)}(\mathbf{u})\right|_{S}=0 \quad \Rightarrow \\
\Rightarrow \quad\left\|\lambda \mathbf{u}-\mathbb{L}_{1}^{(F)}(\mathbf{u})\right\| \geqslant \frac{1}{2}|\lambda|\|\mathbf{u}\| \cos \alpha
\end{gathered}
$$

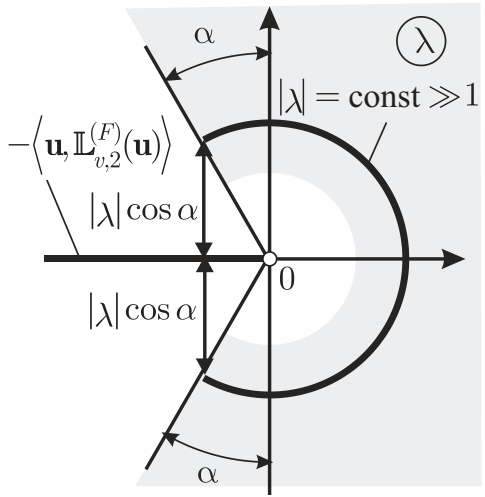

Рис. 3. К оценке нормы

условие (25) выполнено и при $|\lambda| \gg 1,0<\alpha<\pi / 2, \operatorname{Re} \lambda>-|\lambda| \sin \alpha$ согласно теореме 1 характеристический квазимногочлен $D(\lambda)$ и возмущающие квазимногочлены $Q_{k j}(\lambda), k=1,2, \ldots, N_{y}$, $j=1,2, \ldots, N_{x}$, будут аналитическими функциями $\lambda$.

После исключения некоторых вспомогательных величин (например, давления на граничных поверхностях в математических моделях поддерживающих слоев вязкой несжимаемой жидкости [4]) линейный оператор $\mathbb{L}_{1}^{(F)}$ может быть преобразован к виду

$$
\mathbb{L}_{1}^{(F)}=\lambda \mathbb{L}_{v, 0}^{(F)}+\mathbb{L}_{v, 1}^{(F)}-\mathbb{L}_{v, 2}^{(F)} .
$$

Здесь $\mathbb{L}_{v, 0}^{(F)}$ - ограниченный положительно определенный самосопряженный оператор

$$
\begin{gathered}
\forall\left(\mathbf{u}: \Omega \rightarrow \mathbb{R}^{N_{u}}\right):\left.\quad \mathbb{L}_{1}^{(G)}(\mathbf{u})\right|_{S}=0 \quad \Rightarrow \quad 0 \leqslant\left\langle\mathbf{u}, \mathbb{L}_{v, 0}^{(F)}(\mathbf{u})\right\rangle \leqslant C_{0}\|\mathbf{u}\|^{2}, 0<C_{0}<1 \\
\forall\left(\mathbf{u}, \mathbf{v}: \Omega \rightarrow \mathbb{R}^{N_{u}}\right):\left.\quad \mathbb{L}_{1}^{(G)}(\mathbf{u})\right|_{S}=0,\left.\mathbb{L}_{1}^{(G)}(\mathbf{v})\right|_{S}=0 \Rightarrow\left\langle\mathbf{v}, \mathbb{L}_{v, 0}^{(F)}(\mathbf{u})\right\rangle=\left\langle\mathbf{u}, \mathbb{L}_{v, 0}^{(F)}(\mathbf{v})\right\rangle .
\end{gathered}
$$

Поскольку операторы $\mathbb{L}_{v, 0}^{(F)}$ и $\mathbb{L}_{1}^{(G)}$ вещественные, то

$$
\forall\left(\mathbf{u}: \Omega \rightarrow \mathbb{C}^{N_{u}}\right):\left.\quad \mathbb{L}_{1}^{(G)}(\mathbf{u})\right|_{S}=0 \quad \Rightarrow \quad 0 \leqslant\left\langle\mathbf{u}, \mathbb{L}_{v, 0}^{(F)}(\mathbf{u})\right\rangle \leqslant C_{0}\|\mathbf{u}\|^{2}, 0<C_{0}<1 .
$$


Теорема 3. Пусть выполнены условия (22)-(24), (29), (31)-(33). Тогда при $|\lambda| \gg 1, \operatorname{Re} \lambda>$ $>-|\lambda| \sin \alpha, 0<\alpha<\pi / 2$, характеристический квазимногочлен $D(\lambda)$ и возмущающие квазимногочлены $Q_{k j}(\lambda), k=\overline{1, N_{y}}, j=\overline{1, N_{x}}$, - аналитические функции $\lambda$.

Доказательство. Аналогично теореме 2 сводится к выводу неравенства $\forall\left(\mathbf{u}: \Omega \rightarrow \mathbb{C}^{N_{u}}\right)$ : $\left.\mathbb{L}_{1}^{(G)}(\mathbf{u})\right|_{S}=0 \Rightarrow\left\|\lambda \mathbf{u}-\mathbb{L}_{1}^{(F)}(\mathbf{u})\right\| \geqslant \frac{1}{2}\left(1-C_{0}\right)|\lambda|\|\mathbf{u}\| \cos \alpha$.

Пусть теперь рассматривается движение упругой среды с учетом диссипации энергии. Достаточно рассмотреть вспомогательную краевую задачу (26), где

$$
\mathbf{w}(\mathbf{r}, \lambda)=\{\mathbf{u}(\mathbf{r}, \lambda), \lambda \mathbf{u}(\mathbf{r}, \lambda)\}, \quad \mathbb{L}_{1}^{(F)}=\mathbb{L}_{u, 1}^{(F)}-\mathbb{L}_{u, 2}^{(F)}+\lambda\left(\mathbb{L}_{v, 1}^{(F)}-\mathbb{L}_{v, 2}^{(F)}\right),
$$

a $\mathbf{u}(\mathbf{r}, \lambda)$ - собственно упругие смещения частиц среды. Если полагать, что граничные условия накладываются лишь на смещения частиц среды, то (26) принимает вид

$$
\lambda^{2} \mathbf{u}=\mathbb{L}_{u, 1}^{(F)}(\mathbf{u})-\mathbb{L}_{u, 2}^{(F)}(\mathbf{u})+\lambda\left(\mathbb{L}_{v, 1}^{(F)}(\mathbf{u})-\mathbb{L}_{v, 2}^{(F)}(\mathbf{u})\right)+\sum_{j=1}^{2} \lambda^{j} \mathbf{f}_{j}(\mathbf{r}), \quad \mathbf{r} \in \Omega ;\left.\quad \mathbb{L}_{1}^{(G)}(\mathbf{u})\right|_{S}=0 .
$$

Операторы $\mathbb{L}_{v, 1}^{(F)}, \mathbb{L}_{v, 2}^{(F)}$ удовлетворяют свойствам (29)-(31), а $\mathbf{f}_{j}(\mathbf{r})$ вычисляются аналогично (26). Оператор $\mathbb{L}_{u, 1}^{(F)}$ может быть ограничен либо неограничен. Линейный оператор $\mathbb{L}_{u, 2}^{(F)}$ неограничен, положительно определен, самосопряжен и содержит производные наивысшего порядка по независимым пространственным переменным

$$
\begin{aligned}
& \forall\left(\mathbf{u}: \Omega \rightarrow \mathbb{R}^{N_{u}}\right): \quad \mathbf{u} \neq \mathbf{0},\left.\quad \mathbb{L}_{1}^{(G)}(\mathbf{u})\right|_{S}=0 \quad \Rightarrow \quad\left\langle\mathbf{u}, \mathbb{L}_{u, 2}^{(F)}(\mathbf{u})\right\rangle>0, \\
& \forall\left(\mathbf{u}, \mathbf{v}: \Omega \rightarrow \mathbb{R}^{N_{u}}\right):\left.\quad \mathbb{L}_{1}^{(G)}(\mathbf{u})\right|_{S}=0,\left.\quad \mathbb{L}_{1}^{(G)}(\mathbf{v})\right|_{S}=0 \Rightarrow\left\langle\mathbf{v}, \mathbb{L}_{u, 2}^{(F)}(\mathbf{u})\right\rangle=\left\langle\mathbf{u}, \mathbb{L}_{u, 2}^{(F)}(\mathbf{v})\right\rangle .
\end{aligned}
$$

Поскольку операторы $\mathbb{L}_{u, 2}^{(F)}$ и $\mathbb{L}_{1}^{(G)}$ являются вещественными, то

$$
\forall\left(\mathbf{u}: \Omega \rightarrow \mathbb{C}^{N_{u}}\right): \quad\|\mathbf{u}\|=1,\left.\quad \mathbb{L}_{1}^{(G)}(\mathbf{u})\right|_{S}=0 \quad \Rightarrow \quad\left\langle\mathbf{u}, \mathbb{L}_{u, 2}^{(F)}(\mathbf{u})\right\rangle>0
$$

Оператор $\mathbb{L}_{u, 2}^{(F)}$ содержит производные наивысшего порядка, а характерная величина $а$ диссипативных сил относительно упругих является малой, но конечной. Полагаем

$$
\begin{gathered}
\forall\left(\mathbf{u}: \Omega \rightarrow \mathbb{C}^{N_{u}}\right): \quad\|\mathbf{u}\|=1,\left.\quad \mathbb{L}_{1}^{(G)}(\mathbf{u})\right|_{S}=0, \quad\left\|\mathbb{L}_{u, 2}^{(F)}(\mathbf{u})\right\| \gg 1 \quad \Rightarrow \\
\Rightarrow \quad\left|\left\langle\mathbf{u}, \mathbb{L}_{u, 1}^{(F)}(\mathbf{u})\right\rangle\right| \ll\left\langle\mathbf{u}, \mathbb{L}_{u, 2}^{(F)}(\mathbf{u})\right\rangle, \quad\left\langle\mathbf{u}, \mathbb{L}_{v, 2}^{(F)}(\mathbf{u})\right\rangle \approx a\left\langle\mathbf{u}, \mathbb{L}_{u, 2}^{(F)}(\mathbf{u})\right\rangle, \quad a>0 .
\end{gathered}
$$

Теорема 4. Пусть выполнены условия (22)-(24), (29), (31), (35), (37), (39). Тогда при $\lambda \rightarrow \infty$, $\operatorname{Re} \lambda>-\varepsilon, 0<\varepsilon \ll 1$, характеристический квазимногочлен $D(\lambda)$ и возмущающие квазимногочлены $Q_{k j}(\lambda), k=\overline{1, N}_{y}, j=\overline{1, N_{x}},-$ аналитические функщии параметра $\lambda$.

Доказательство. Достаточно рассмотреть линейную краевую задачу (36), для решения которой при $\lambda \rightarrow \infty, \operatorname{Re} \lambda>-\infty$ из $\|\mathbf{u}\|<M<\infty$ следует $\left\|\mathbb{L}_{u, 2}^{(F)}(\mathbf{u})\right\| \rightarrow \infty,\left\|\mathbb{L}_{v, 2}^{(F)}(\mathbf{u})\right\| \rightarrow \infty$. Как следует из (30), (31), при $\lambda \rightarrow \infty, \operatorname{Re} \lambda>-\varepsilon, 0<\varepsilon \ll 1$

$$
\begin{gathered}
\forall\left(\mathbf{u}: \Omega \rightarrow \mathbb{C}^{N_{u}}\right): \quad\|\mathbf{u}\|=1,\left.\quad \mathbb{L}_{1}^{(G)}(\mathbf{u})\right|_{S}=0 \quad \Rightarrow \\
\Rightarrow \quad\left\|\lambda^{2} \mathbf{u}-\left[\mathbb{L}_{u, 1}^{(F)}(\mathbf{u})-\mathbb{L}_{u, 2}^{(F)}(\mathbf{u})+\lambda\left(\mathbb{L}_{v, 1}^{(F)}(\mathbf{u})-\mathbb{L}_{v, 2}^{(F)}(\mathbf{u})\right)\right]\right\| \geqslant \\
\geqslant\left|\left\langle\mathbf{u}, \lambda^{2} \mathbf{u}-\left[\mathbb{L}_{u, 1}^{(F)}(\mathbf{u})-\mathbb{L}_{u, 2}^{(F)}(\mathbf{u})+\lambda\left(\mathbb{L}_{v, 1}^{(F)}(\mathbf{u})-\mathbb{L}_{v, 2}^{(F)}(\mathbf{u})\right)\right]\right\rangle\right| \geqslant \\
\geqslant \frac{1}{2}\left|\lambda^{2}+\lambda\left\langle\mathbf{u}, \mathbb{L}_{v, 2}^{(F)}(\mathbf{u})\right\rangle+\left\langle\mathbf{u}, \mathbb{L}_{u, 2}^{(F)}(\mathbf{u})\right\rangle\right| \geqslant \frac{1}{4}|P(\lambda)|>0, \\
P(\lambda)=\lambda^{2}+\lambda a\left\langle\mathbf{u}, \mathbb{L}_{u, 2}^{(F)}(\mathbf{u})\right\rangle+\left\langle\mathbf{u}, \mathbb{L}_{u, 2}^{(F)}(\mathbf{u})\right\rangle .
\end{gathered}
$$


поскольку при $a>0$ корни полинома $P(\lambda)$ второй степени под знаком модуля имеют строго отрицательные вещественные части, т. е. при $\lambda \rightarrow \infty$, $\operatorname{Re} \lambda>-\varepsilon, 0<\varepsilon \ll 1$

$$
\begin{gathered}
\forall\left(\mathbf{u}: \Omega \rightarrow \mathbb{C}^{N_{u}}\right):\left.\quad \mathbb{L}_{1}^{(G)}(\mathbf{u})\right|_{S}=0 \quad \Rightarrow \\
\Rightarrow \quad\left\|\lambda^{2} \mathbf{u}-\left[\mathbb{L}_{u, 1}^{(F)}(\mathbf{u})-\mathbb{L}_{u, 2}^{(F)}(\mathbf{u})+\lambda\left(\mathbb{L}_{v, 1}^{(F)}(\mathbf{u})-\mathbb{L}_{v, 2}^{(F)}(\mathbf{u})\right)\right]\right\| \geqslant \frac{1}{4}|P(\lambda)|\|\mathbf{u}\|, \quad|P(\lambda)|>0
\end{gathered}
$$

аналог условия (25) выполнен, и согласно теореме $1 D(\lambda)$ и $Q_{k j}(\lambda), k=\overline{1, N_{y}}, j=\overline{1, N_{x}}$, будут аналитическими функциями $\lambda$ при $|\lambda| \gg 1$, $\operatorname{Re} \lambda>-\varepsilon, 0<\varepsilon \ll 1$.

Замечание 1. Если учесть, что, как правило, при $\lambda \rightarrow \infty\left\langle\mathbf{u}, \mathbb{L}_{u, 2}^{(F)}(\mathbf{u})\right\rangle \rightarrow \infty$, то оба корня полинома $P(\lambda)$ будут вещественные строго отрицательные, причем ближайший к точке $\lambda=0$ корень $\approx 1 / a$. В этом случае при $\lambda \rightarrow \infty, 0<\alpha<\pi / 2, \operatorname{Re} \lambda>-|\lambda| \sin \alpha|P(\lambda)| \geqslant|\lambda|^{2} \cos ^{2} \alpha$, и, следовательно, характеристический квазимногочлен $D(\lambda)$ и возмущающие квазимногочлены $Q_{k j}(\lambda)$, $k=\overline{1, N_{y}}, j=\overline{1, N_{x}}$, будут аналитическими функциями $\lambda$ при $|\lambda| \gg 1,0<\alpha<\pi / 2, \operatorname{Re} \lambda>-|\lambda| \sin \alpha$.

\section{5. ПРИМЕРЫ ПРИМЕНЕНИЯ}

В качестве примера применения теоремы 3 можно привести нелинейную комбинированную динамическую модель цилиндрического гидродинамического подвеса

$$
\begin{gathered}
\pi \beta\left(\frac{\rho_{2}}{\rho}-1\right) \ddot{\mathbf{y}}=\pi\left(\frac{\rho_{2}}{\rho}-1\right) \gamma(\mathbf{g}-\mathbf{a})+\mathbf{N}, \quad \pi \frac{\rho_{2}}{\rho} J \dot{\omega}=-\frac{\beta}{\sigma} G, \quad \mathbf{g}=(0,-1)^{T} \\
h(\varphi, t)=1-\mathbf{y} \cdot \mathbf{e}_{r}-\frac{1}{2} \beta\left(\mathbf{y} \cdot \mathbf{e}_{\varphi}\right)^{2}, \quad p=p(0, \varphi, t)+\beta \int_{0}^{\xi}\left(v_{\varphi}^{2}+2 v_{\varphi}\right) d \xi \\
v_{r}=-(1-\beta \xi) \int_{0}^{\xi} \frac{\partial v_{\varphi}}{\partial \varphi} d \xi, \quad \frac{\partial}{\partial \varphi} \int_{0}^{h} v_{\varphi} d \xi=\frac{\partial}{\partial \varphi}\left[(1+\beta h)\left(\mathbf{y} \cdot \mathbf{e}_{r}-\dot{\mathbf{y}} \cdot \mathbf{e}_{\varphi}\right)\right] \\
\frac{\partial v_{\varphi}}{\partial t}+\left[1+(1-\beta \xi) v_{\varphi}\right] \frac{\partial v_{\varphi}}{\partial \varphi}+v_{r} \frac{\partial v_{\varphi}}{\partial \xi}+\beta v_{r}\left(2+v_{\varphi}\right)= \\
=-(1-\beta \xi) \frac{\partial p}{\partial \varphi}+\frac{1}{\sigma}\left(\frac{\partial^{2} v_{\varphi}}{\partial \xi^{2}}+\beta \frac{\partial v_{\varphi}}{\partial \xi}+\beta^{2} \frac{\partial^{2} v_{\varphi}}{\partial \varphi^{2}}\right) \\
v_{\varphi}(0, \varphi, t)=-\omega, \quad v_{\varphi}(h, \varphi, t)=\beta\left(\mathbf{y} \cdot \mathbf{e}_{r}-\dot{\mathbf{y}} \cdot \mathbf{e}_{\varphi}\right) \\
\int_{0}^{2 \pi}\left(\frac{\beta}{\sigma} \frac{\partial v_{\varphi}(0, \varphi, t)}{\partial \xi}-\frac{\partial p(0, \varphi, t)}{\partial \varphi}\right) \mathbf{e}_{\varphi} d \varphi, \quad G=2 \pi \beta \omega+\int_{0}^{2 \pi} \frac{\partial v_{\varphi}(0, \varphi, t)}{\partial \xi} d \varphi \\
\mathbf{e}_{r}=(\cos \varphi, \sin \varphi)^{T}, \quad \mathbf{e}_{\varphi}=(-\sin \varphi, \cos \varphi)^{T},
\end{gathered}
$$

отличающуюся от [4] лишь малым слагаемым $\beta^{2} \partial^{2} v_{\varphi} / \partial \varphi^{2}$ в укороченном уравнении Навье - Стокса. Здесь $\mathbf{y} \in \mathbb{R}^{2}$ - координаты центра масс внутреннего тела, $\mathbf{r}=(\xi, \varphi)^{T}$ - безразмерные радиальная координата и полярный угол (независимые пространственные координаты), $v_{r}, v_{\varphi}, p$ - безразмерные радиальная, тангенциальная компоненты скорости и давление в поддерживающем слое соответственно. Выбор безразмерных переменных и параметров обоснован в [4]. Теорема 3 гарантирует для математической модели (40) аналитичность в высокочастотной области характеристического и возмущающих квазимногочленов (вычисляются аналогично [4]). Если учесть, что в соответствии с [4] для линеаризованных уравнений возмущенного движения в изображениях Лапласа в силу периодичности решения по координате $\varphi$ при $\lambda \rightarrow \infty, \operatorname{Re} \lambda>-\infty$, в пределах пограничных слоев $\left|\partial v_{\varphi} / \partial \xi\right| \gg\left|\partial v_{\varphi} / \partial \varphi\right|$, то становится обоснованным применение теоремы 3 к математической модели [4]. Детальное исследование устойчивости цилиндрического гидродинамического подвеса на основе критерия устойчивости (14) ранее было выполнено в [4]. Равновесное состояние моделировалось на основе проекционного метода Галеркина в форме (15)-(17), в низкочастотной области численное решение вспомогательных линейных краевых задач было реализовано на основе проекционного метода Галеркина (18), (19), а в высокочастотной области применялось асимптотическое интегрирование на основе метода сращиваемых разложений. 
В качестве примера применения теоремы 4 можно привести статью [5], где исследована устойчивость и выполнен параметрический синтез системы угловой стабилизации подвижного объекта управления. После приведения к безразмерным переменным и параметрам линеаризованные модельные уравнения движения КДС принимают вид [5]

$$
\begin{gathered}
J_{0} \ddot{\beta}_{0}=-p_{1} \dot{\beta}_{0}-p_{2} \beta_{0}+\mathbb{S}\left(\beta_{1}+\beta_{2}\right), \quad m_{1} \ddot{y}_{1}=\left(1+m_{1}+m_{2}\right) \beta_{0}+P_{1}-F_{e}, \\
J_{0} \ddot{\beta}_{0}+J_{1} \ddot{\beta}_{1}=M_{1}, \quad m_{2}\left[(1+a) \ddot{\beta}_{1}+\ddot{y}_{1}+\ddot{y}_{2}\right]=P_{2}+a_{x} m_{2} \beta_{2}, \\
J_{2}\left(\ddot{\beta}_{1}+\ddot{\beta}_{2}\right)=M_{2}-a P_{2}, \quad \mathbb{S}(.)=p_{3} d(.) / d t+p_{4} \cdot(.)+p_{5} \int_{0}^{t}(.) d t, \\
\ddot{u}+u^{\prime \prime \prime \prime}+\gamma \dot{u}^{\prime \prime \prime \prime}+a_{x}\left[\left(m_{2}+(1-x)\right) u^{\prime}\right]^{\prime}=-\ddot{y}_{1}-x \ddot{\beta}_{1}, \quad(.)^{\prime}=\partial(.) / \partial x, \\
u(0, t)=0, \quad u^{\prime}(0, t)=0, \quad u(1, t)=y_{2}(t), \quad u^{\prime}(1, t)=\beta_{2}(t), \\
M_{1}=u^{\prime \prime}(0, t)+\gamma \dot{u}^{\prime \prime}(0, t), \quad P_{1}=-u^{\prime \prime \prime}(0, t)-\gamma \dot{u}^{\prime \prime \prime}(0, t), \\
M_{2}=-u^{\prime \prime}(1, t)-\gamma \dot{u}^{\prime \prime}(1, t), \quad P_{2}=u^{\prime \prime \prime}(1, t)+\gamma \dot{u}^{\prime \prime \prime}(1, t), \\
\beta_{0}(0)=\beta_{1}(0)=\beta_{2}(0)=\dot{\beta}_{0}(0)=\dot{\beta}_{1}(0)=\dot{\beta}_{2}(0)=y_{1}(0)=y_{2}(0)=\dot{y}_{1}(0)=\dot{y}_{2}(0)=0, \\
u(x, 0)=\dot{u}(x, 0)=0 .
\end{gathered}
$$

Здесь выходная вектор-функция $\mathbf{y}(t)=\left(\beta_{1}(t), \beta_{2}(t)\right)^{T}$ характеризует ошибки системы стабилизации, а параметры обратных связей $\mathbf{p}=\left(p_{1}, p_{2}, p_{3}, p_{4}, p_{5}\right)^{T}$ требуется выбрать так, чтобы наиболее эффективно подавлять ошибку системы стабилизации. Для математической модели (41) аналитичность в высокочастотной области характеристического и возмущающих квазимногочленов (см. [5]) гарантируется теоремой 4. Численное решение вспомогательных краевых задач в низкочастотной области выполнялось на основе проекционного метода Галеркина (18), (19), а в высокочастотной области применялось асимптотическое интегрирование на основе ВКБ-приближения. При этом проверка устойчивости на основе (14) выполнялась настолько быстро, что это не препятствовало распараллеливанию параметрического синтеза [5] на основе классических технологий параллельного программирования ОpenMP и MPI.

Аналогичный подход ранее использован в [6], где аналитичность в высокочастотной области характеристического и возмущающих квазимногочленов также гарантируется теоремой 4, а дальнейшее распараллеливание параметрического синтеза семейства линеаризованных КДС в [7] выполнялось на основе стандартных средств технологии параллельного программирования Microsoft Concurrency Runtime.

\section{Библиографиический список}

1. Андрейченко Д. К., Андрейченко К. П. К теории комбинированных динамических систем // Изв. РАН. Теория и системы управления. 2000. № 3. C. 54-69.

2. Андрейченко Д. К., Андрейченко К. П. Моделирование, анализ и синтез комбинированных динамических систем : учеб. пособие. Саратов : РайтЭкспо, 2013. 144 с.

3. Люстерник Л. А., Соболев В. И. Краткий курс функционального анализа. М. : Высш. шк., 1982. $271 \mathrm{c.}$

4. Андрейченко Д. К., Андрейченко К. П. К теории устойчивости цилиндрического гидродинамического подвеса // Изв. РАН. Механика жидкости и газа. 2009. № 1. С. 13-26.

5. Андрейченко Д. К., Ерофтиев А. А., Мельничук Д. В. Распараллеливание параметрического синтеза по схеме «Портфель задач» на основе технологии MPI // Изв. Сарат. ун-та. Нов. сер. Сер. Математика. Механика. Информатика. 2015. T. 15, вып. 2. С. 222-228. DOI: 10.18500/18169791-2015-15-2-222-228.

6. Андрейченко Д. К., Андрейченко К. П., Кононов В. В. К теории устойчивости автономной системы угловой стабилизации реактивного снаряда залпового огня // Изв. Сарат. ун-та. Нов. сер. Сер. Математика. Механика. Информатика. 2013. T. 13, вып. 2, ч. 2. С. 9-14.

7. Андрейченко Д. К., Андрейченко К. П., Кононов В. В. Параллельный алгоритм вычисления оптимальных параметров одноканальной системы угловой стабилизации // Изв. Сарат. ун-та. Нов. сер. Сер. Математика. Механика. Информатика. 2013. Т. 13, вып. 4, ч. 1. С. 109-117. 


\title{
Analyticity Conditions of Characteristic and Disturbing Quasipolynomials of Hybrid Dynamical Systems
}

\author{
M. S. Portenko ${ }^{1}$, D. V. Melnichuk ${ }^{2}$, D. K. Andreichenko ${ }^{3}$
}

\begin{abstract}
${ }^{1}$ Marina S. Portenko, Saratov State University, 83, Astrakhanskaya st., 410012, Saratov, Russia, msportenko@gmail.com ${ }^{2}$ Dmitry V. Melnichuk, Saratov State University, 83, Astrakhanskaya st., 410012, Saratov, Russia, meldm007@ gmail.com ${ }^{3}$ Dmitry K. Andreichenko, Saratov State University, 83, Astrakhanskaya st., 410012, Saratov, Russia, kp_andreichenko@ renet.ru Hybrid dynamical systems (HDS) are connected by means of the boundary conditions and the constraint's conditions systems of ordinary differential equations and partial differential equations with the corresponding initial conditions. Check the stability of HDS can be performed on the basis of the "fast"algorithm for the application which requires analytic characteristic and disturbing quasipolynomials of HDS in the right half-plane and near the imaginary axis. In this paper we formulate and prove the analyticity conditions of the characteristic and disturbing HDS quasipolynomials. Mathematical models of control objects with distributed parameters in space, matching the thermal conductivity and diffusion processes, the dynamics of support layers of viscous incompressible fluid, as well as the dynamics of the elastically deformable medium taking into account the internal friction.
\end{abstract}

Key words: hybrid dynamical systems, stability.

\section{References}

1. Andreichenko D. K., Andreichenko K. P. On the theory of hybrid dynamical systems. Journal of Computer and Systems Sciences International, 2000, vol. 39, no 3, pp. 383-398.

2. Andreichenko D. K., Andreichenko K. P. Modelirovanie, analiz $i$ sintez kombinirovannykh dinamicheskikh sistem. Uchebnoe posobie [Modeling, analysis and synthesis of combined dynamical systems. Tutorial]. Saratov, Rait-Ekspo, 2013, 144 p. (in Russian).

3. Liusternik L. A., Sobolev V. I. Kratkii kurs funktsional'nogo analiza [A short course of functional analysis]. Moscow, Vysshaia shkola, 1982, 271 p. (in Russian).

4. Andreichenko D. K., Andreichenko K. P. On the theory of stability of a cylindrical hydrodynamic suspension. Fluid Dynamics, 2009, vol. 44, no 1, pp. $10-21$.

5. Andreichenko D. K., Eroftiev A. A., Melni- chuk D. V. Parallelization of parametric synthesis by "problems portfolio"scheme based on MPI technology. Izv. Saratov Univ. (N. S.), Ser. Math. Mech. Inform., 2015, vol. 15, no. 2, pp. 222-228 (in Russian). DOI: 10.18500/1816-9791-2015-15-2222-228.

6. Andreichenko D. K., Andreichenko K. P., Kononov V. V. On stability theory of autonomous angular stabilization system for combined dynamical systems. Izv. Saratov Univ. (N. S.), Ser. Math. Mech. Inform., 2013, vol. 13, iss. 2, pt. 2, pp. 9-14 (in Russian).

7. Andreichenko D. K., Andreichenko K. P., Kononov V. V. Parallel algorithm of optimal parameters calculation for the single channel angular stabilization system. Izv. Saratov Univ. (N. S.), Ser. Math. Mech. Inform., 2013, vol. 13, iss. 4, pt. 1, pp. 109117 (in Russian).

УДК 519.72

\section{О ПРИМЕНЕНИИ ВЕЙВЛЕТОВ К ЦИФРОВОЙ ОБРАБОТКЕ СИГНАЛОВ}

\section{Е. А. Родионов}

Родионов Евгений Анатольевич, аспирант кафредры математики, Российский государственный геологоразведочный университет им. Серго Орджоникидзе, Москва, evgeny_980@list.ru

Дискретное вейвлет-преобразование, ассоциированное с фрункциями Уолша, определено Лэнгом (W. C. Lang) в 1998 г. B статье излагаются применения преобразования Лэнга и некоторых его модисрикаций для анализа сринансовых временных рядов и для сжатия сррактальных данных. Показано, что для обработки некоторых сигналов изучаемые дискретные вейвлетпреобразования имеют преимущества по сравнению с дискретными преобразованиями Хаара, Добеши и методом зонного кодирования.

Ключевые слова: цифровая обработка сигналов, вейвлеты, функции Уолша, фиинансовые временные ряды.

DOI: 10.18500/1816-9791-2016-16-2-217-225 